Supporting information for

\title{
Identification of Degradation Products of Sea-Dumped Chemical Warfare Agent-Related Phenylarsenic Chemicals in Marine Sediment
}

Hanna Niemikoski*1, Martin Söderström ${ }^{1}$, Harri Kiljunen ${ }^{1}$, Anders Östin ${ }^{2}$, and Paula Vanninen ${ }^{1}$

Corresponding author: hanna.niemikoski@helsinki.fi

${ }^{1}$ Finnish Institute for Verification of the Chemical Weapons Convention, VERIFIN, Department of Chemistry, P.O. Box 55, FI-00014 University of Helsinki, Finland

${ }^{2}$ Swedish Defence Research Agency, FOI, SE-90182 Umeå, Sweden

\section{TABLE OF Contents}

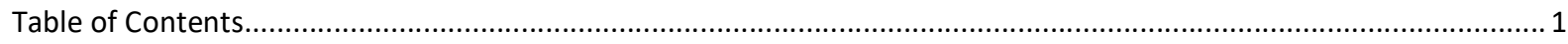

Table S-1 List of current target phenylarsenic CWAs in sediment analysis ................................................................. 2

Table S-2 Concentrations of current target chemicals detected in sediment samples quantitated in CHEMSEA, MODUM

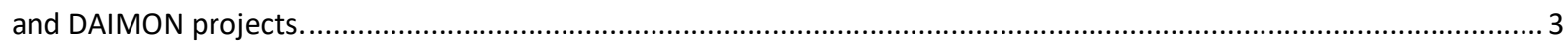

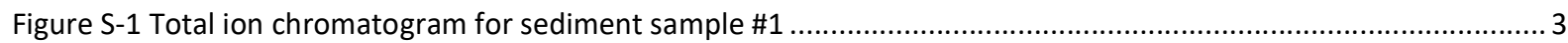

Figure S-2 Extracted ion chromatograms for sediment sample \#6 containing methylated phenylarsenicals and target

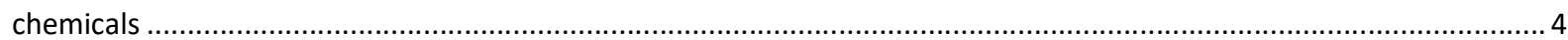

Figure S-3 Total ion chromatogram and spectrum for diphenylthioarsinic acid .................................................. 5

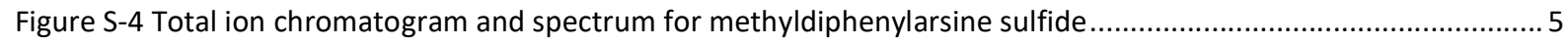

Figure S-5 Total ion chromatogram and spectrum for 10 -methyl-5H-phenarsazinine-10-sulfide ............................... 6

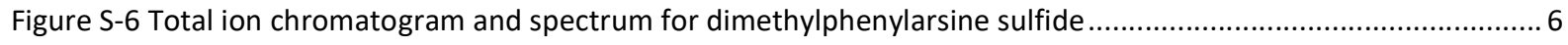

Figure S-7 Total ion chromatogram and spectrum for triphenylarsine sulfide ................................................... 7

Table S-3 Detected fragments and their relative abundance for all detected sulfur containing chemicals.................... 8

Figure S-8 Extracted ion chromatogram for sediment sample \#8 containing sulfur-containing phenylarsenic chemicals

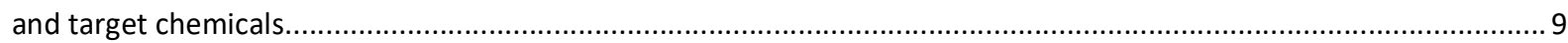

Figure S-9 Extracted ion chromatogram for sediment sample \#8 containing sulfur-containing phenylarsenic chemical

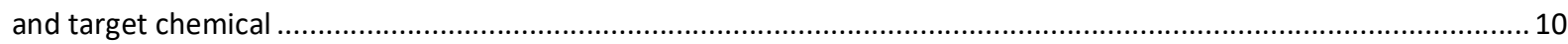

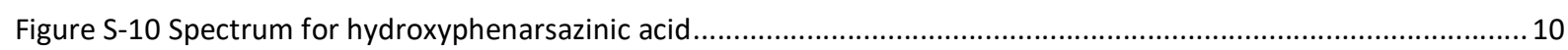

Figure S-11 Extracted ion chromatogram and spectra for two isomers of hydroxydiphenylarsinic acid ...................... 11

Figure S-12 Extracted ion chromatogram and spectra for two isomers of hydroxytriphenylarsine oxide....................12 
TABLE S-1 LIST OF CURRENT TARGET PHENYLARSENIC CWAS IN SEDIMENT ANALYSIS

\begin{tabular}{|c|c|}
\hline Chemical (acronym) & Structure \\
\hline \multicolumn{2}{|l|}{$\begin{array}{l}\text { Adamsite } \\
\text { (DM) }\end{array}$} \\
\hline \multicolumn{2}{|l|}{$\begin{array}{l}\text { 5,10-Dihydrophen- } \\
\text { arsazin-10-ol 10-oxide }\end{array}$} \\
\hline $\begin{array}{l}\text { Clark I } \\
\text { (DA) }\end{array}$ & \\
\hline \multicolumn{2}{|l|}{$\begin{array}{l}\text { Diphenylarsinic acid } \\
\text { (DPA) }\end{array}$} \\
\hline \multicolumn{2}{|l|}{$\begin{array}{l}\text { Triphenylarsine } \\
\text { (TPA) }\end{array}$} \\
\hline \multicolumn{2}{|l|}{$\begin{array}{c}\text { Triphenylarsine oxide } \\
\text { (TPAO) }\end{array}$} \\
\hline $\begin{array}{c}\text { Phenylarsonic acid } \\
\text { (PAA) }\end{array}$ & \\
\hline
\end{tabular}


TABLE S-2 CONCENTRATIONS OF CURRENT TARGET CHEMICALS DETECTED IN SEDIMENT SAMPLES QUANTITATED IN CHEMSEA, MODUM AND DAIMON PROJECTS.

\begin{tabular}{|c|c|c|c|c|c|}
\hline \multirow[b]{2}{*}{$\begin{array}{c}\text { Sample } \\
\text { ID }\end{array}$} & \multirow[b]{2}{*}{ Project } & \multicolumn{4}{|c|}{ concentration $[\mu \mathrm{g} / \mathrm{kg}]$ in dry in weight } \\
\hline & & DMox & DPA & TPAO & PAA \\
\hline$\# 1$ & CHEMSEA & 22 & 1300 & 190 & N.A.* \\
\hline \#2 & CHEMSEA & 5.2 & 1300 & 180 & N.A.* \\
\hline \#3 & CHEMSEA & 30 & 210 & 67 & N.A.* \\
\hline \#4 & MODUM & 3.4 & 15 & 1900 & 120 \\
\hline \#5 & MODUM & N.D. $* *$ & N.D.** & N.D.** & N.D.** \\
\hline \#6 & DAIMON & $79 \pm 30$ & 120 & 300 & 180 \\
\hline \#7 & DAIMON & N.D.** & N.D.** & N.D.** & N.D.** \\
\hline$\# 8$ & DAIMON & 12 & 83 & 310 & 246 \\
\hline \#9 & DAIMON & 151 & 1100 & 195 & 2641 \\
\hline$\# 10$ & CHEMSEA & 37 & 2104 & 310 & 37 \\
\hline$\# 11$ & CHEMSEA & 9.2 & 2302 & 321 & 9.2 \\
\hline$\# 12$ & CHEMSEA & 53 & 369 & 121 & 53 \\
\hline$\# 13$ & DAIMON & 13 & 86 & 321 & 255 \\
\hline$\# 14$ & DAIMON & 144 & 95 & 138 & 191 \\
\hline
\end{tabular}

*N.A. $=$ not analysed, N.D. $=$ not detected

\section{FIGURE S-1 TOTAL ION CHROMATOGRAM FOR SEDIMENT SAMPLE \#1}

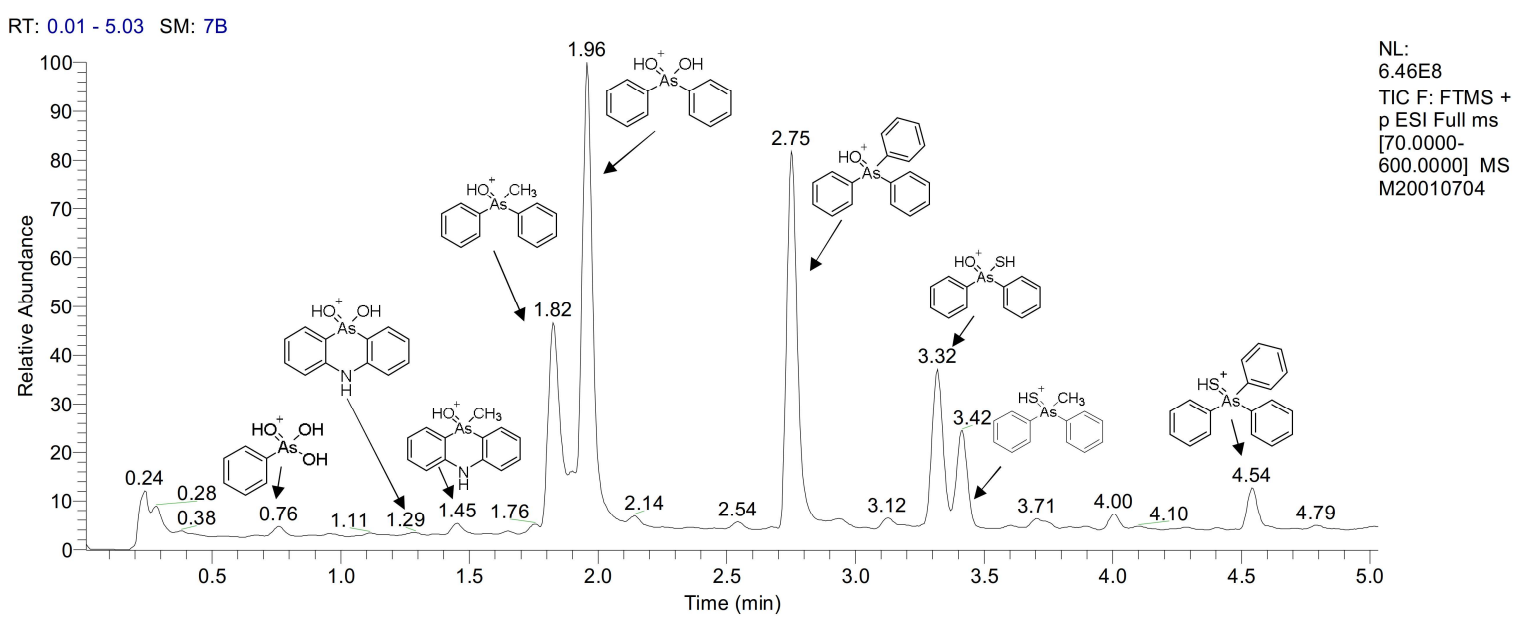


FIGURE S-2 EXTRACTED ION CHROMATOGRAMS FOR SEDIMENT SAMPLE \#6 CONTAINING METHYLATED PHENYLARSENICALS AND TARGET CHEMICALS

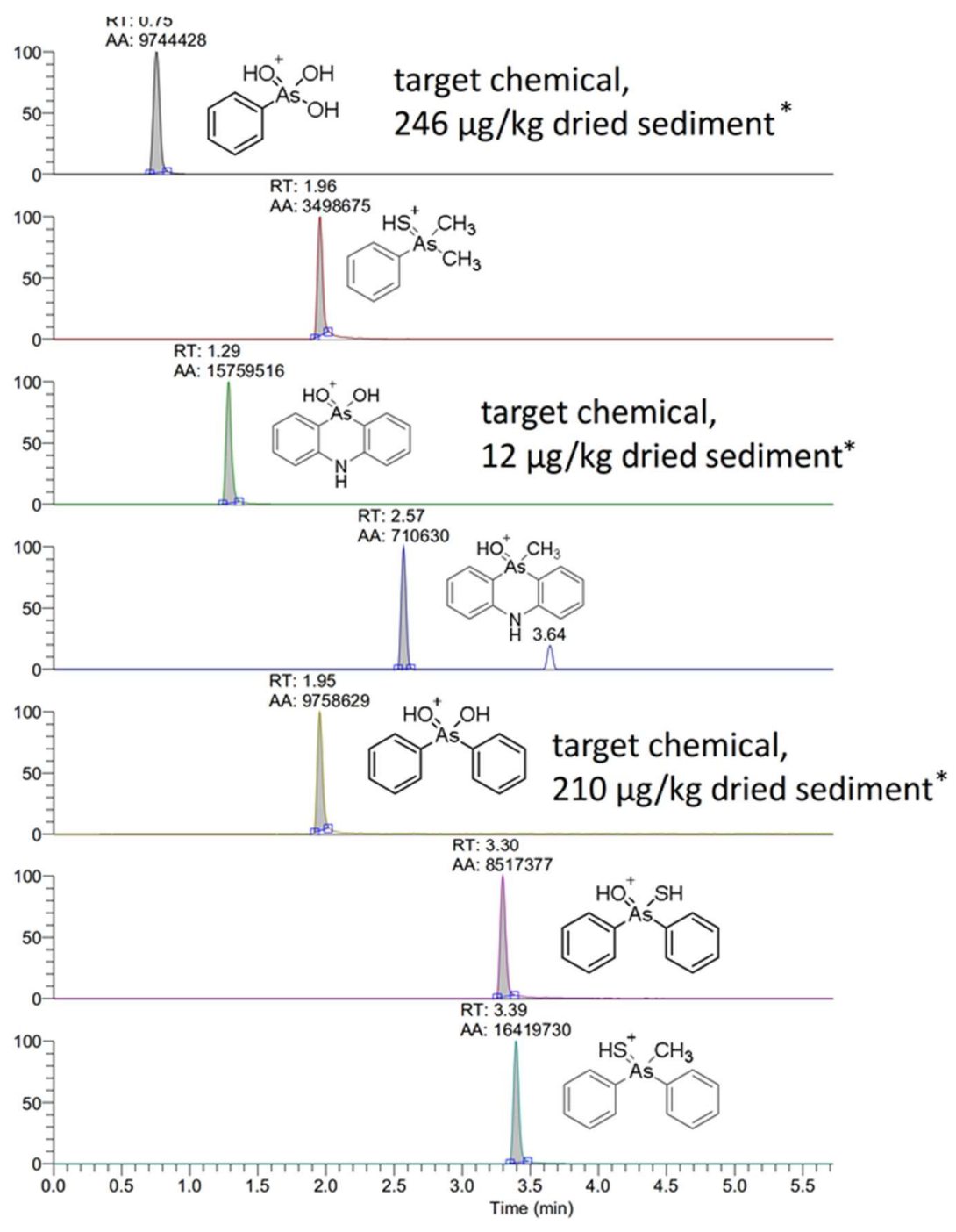

* Concentrations of target chemicals have been quantified previously using LC-MS/MS method from the same sediment sample. 
FIGURE S-3 TOTAL ION CHROMATOGRAM AND SPECTRUM FOR DIPHENYLTHIOARSINIC ACID

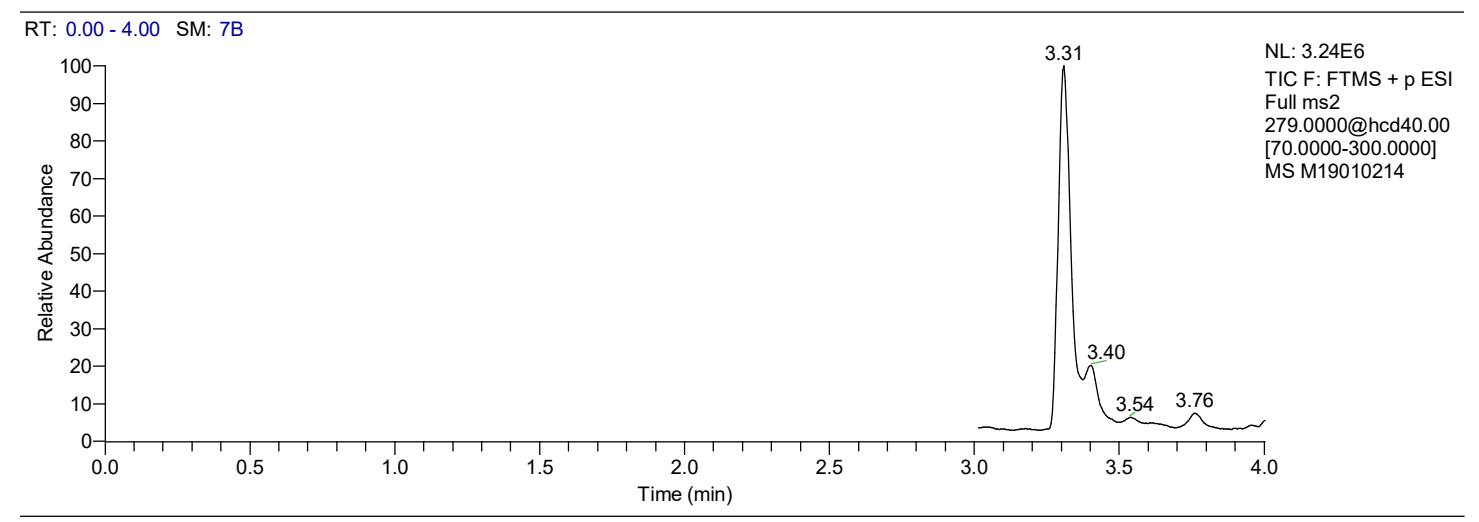

M19010214 \#67 RT: 3.31 AV: 1 SB: 2 3.01, 3.01 NL: 2.09E6

F: FTMS + p ESI Full ms2 279.0000@hcd40.00 [70.0000-300.0000]

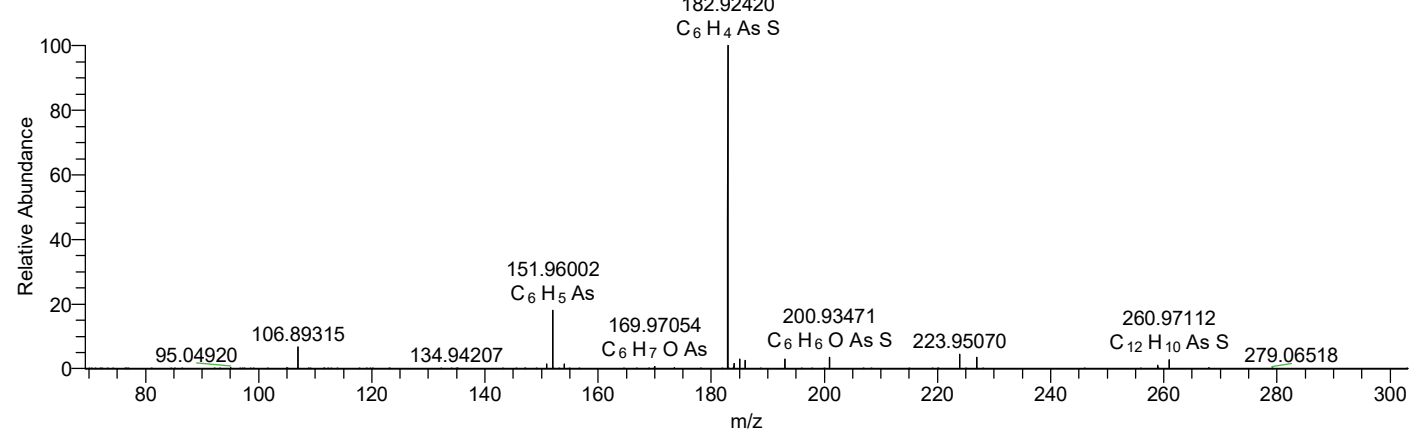

FIGURE S-4 TOTAL ION CHROMATOGRAM AND SPECTRUM FOR METHYLDIPHENYLARSINE SULFIDE

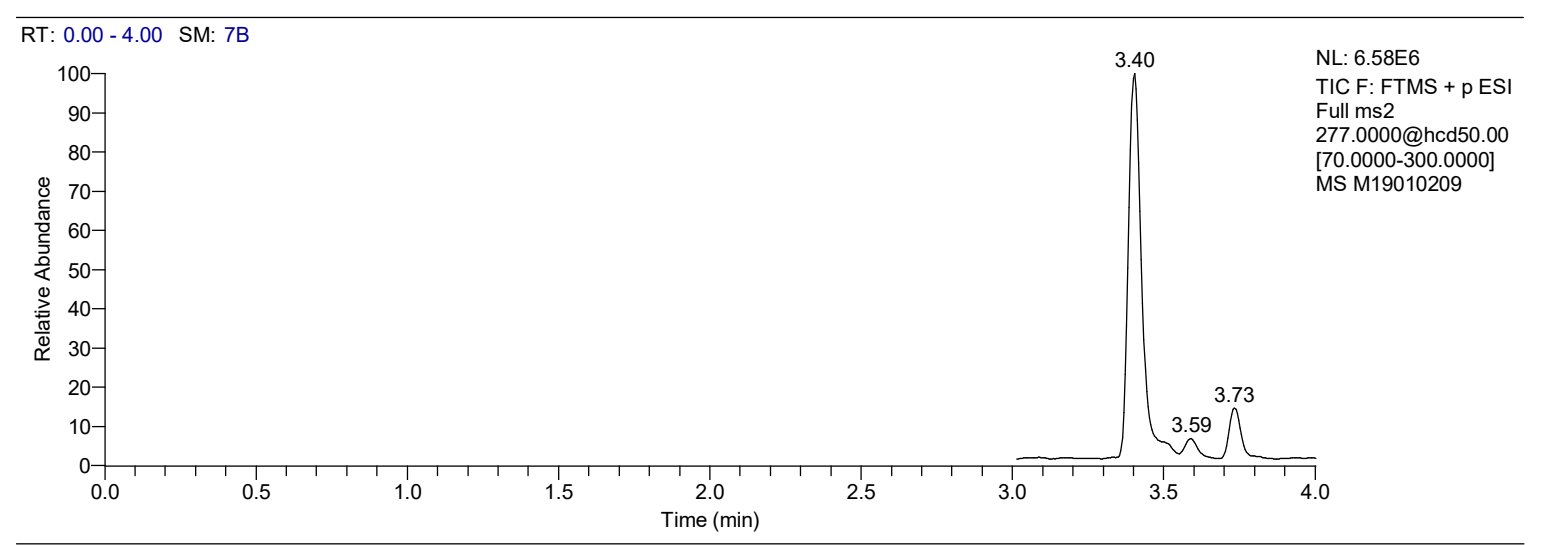

M19010209 \#87 RT: $3.40 \quad$ AV: 1 SB: $23.01,3.01$ NL: 1.40E6

F: FTMS + p ESI Full ms2 277.0000@hcd50.00 [70.0000-300.0000]

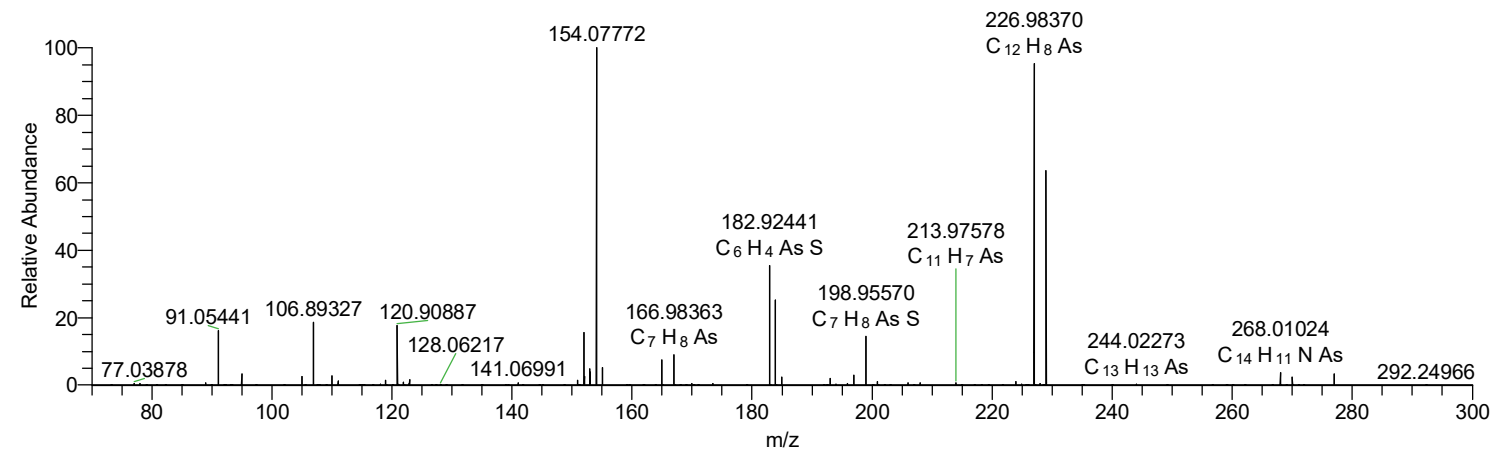


FIgURE S-5 TOTAL ION CHROMATOGRAM AND SPECTRUM FOR 10-METHYL-5H-PHENARSAZININE10-SULFIDE

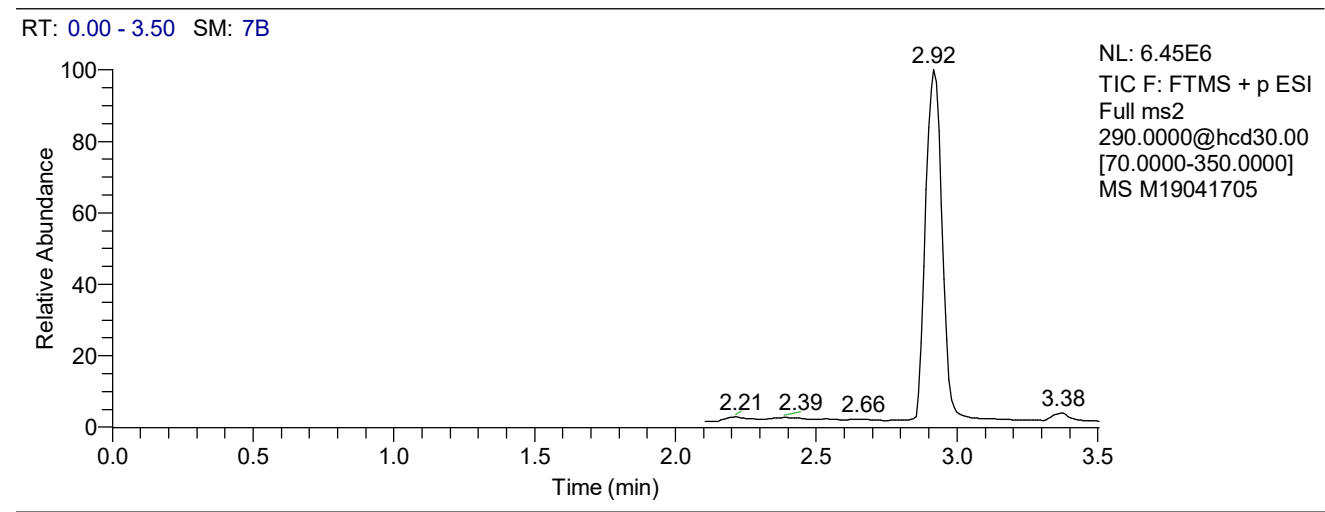

M19041705 \#178 RT: $2.90 \quad$ AV: 1 SB: 75 2.11-2.39, 2.59-2.97 NL: 4.41E6

F: FTMS + p ESI Full ms2 290.0000@hcd30.00 [70.0000-350.0000]

241.99452

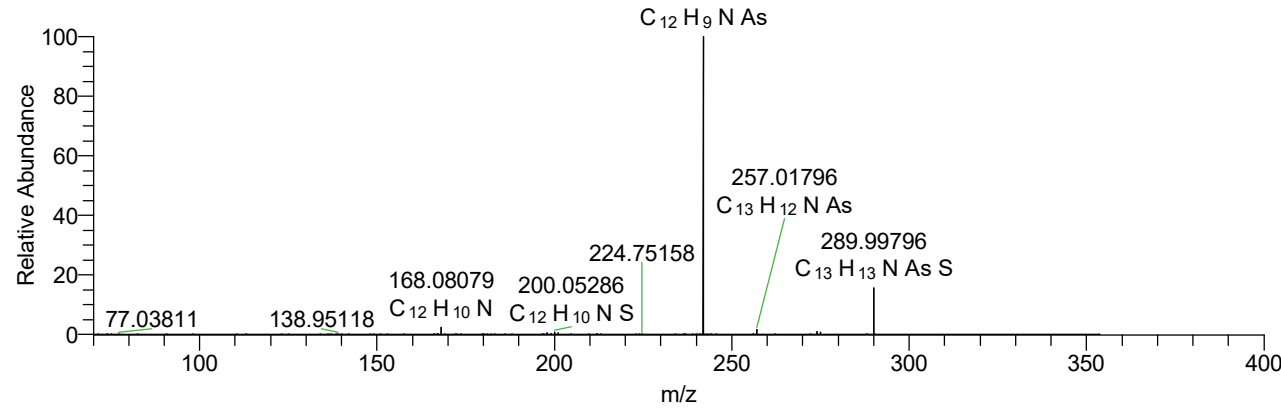

FIGURE S-6 TOTAL ION CHROMATOGRAM AND SPECTRUM FOR DIMETHYLPHENYLARSINE SULFIDE

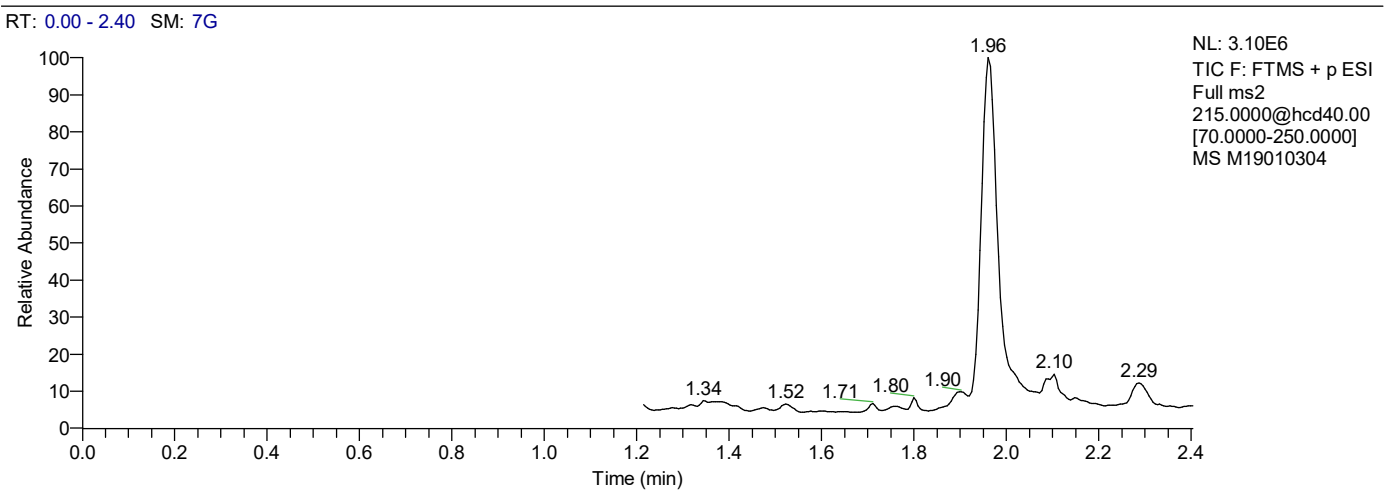

M19010304 \#167 RT: $1.96 \quad$ AV: 1 NL: 1.15E6

F: FTMS + p ESI Full ms2 215.0000@hcd40.00 [70.0000-250.0000]

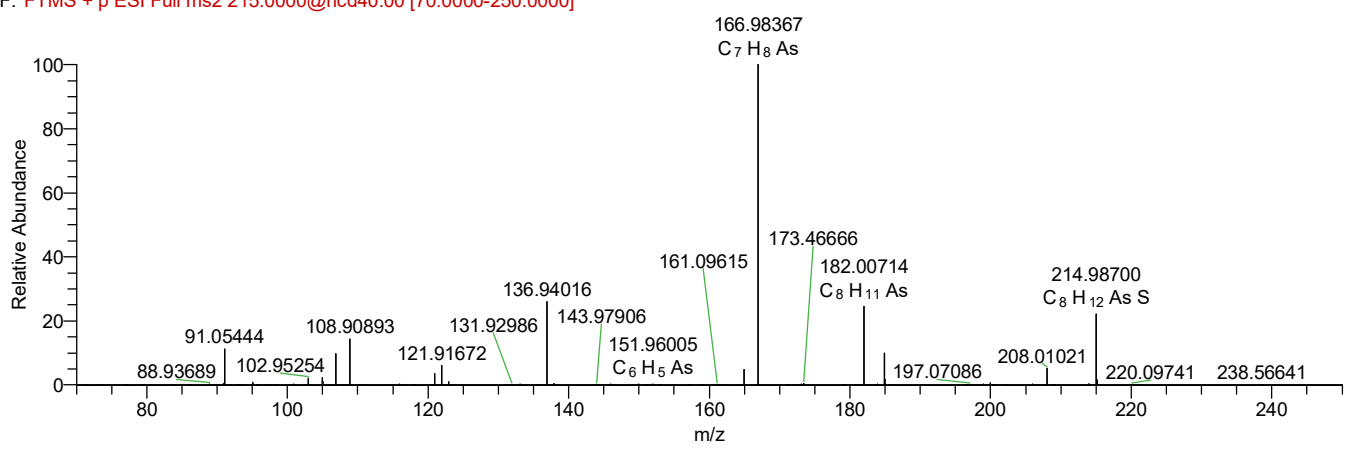


FIGURE S-7 TOTAL ION CHROMATOGRAM AND SPECTRUM FOR TRIPHENYLARSINE SULFIDE

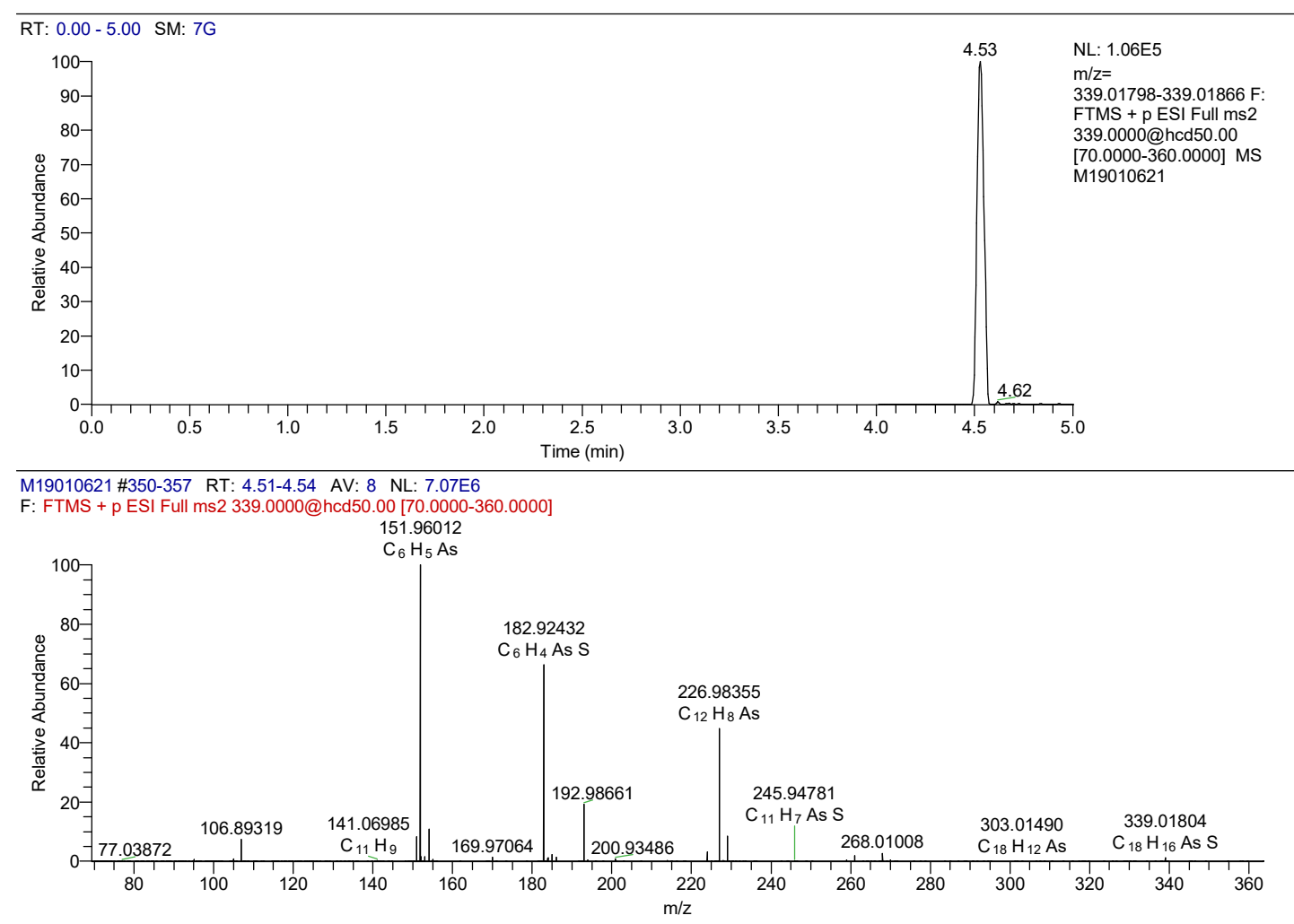




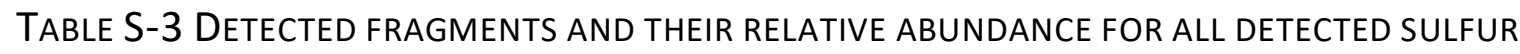
CONTAINING CHEMICALS

\begin{tabular}{|c|c|c|c|}
\hline $\begin{array}{l}\text { Fragment ion } \\
{[\mathrm{m} / \mathrm{z}]}\end{array}$ & Relative abundance [\%] & Fragment ion $[\mathrm{m} / \mathrm{z}]$ & Relative abundance [\%] \\
\hline \multicolumn{2}{|c|}{ Diphenylarsinothio acid } & \multicolumn{2}{|c|}{ Methyldiphenylarsine sulfide } \\
\hline 260.97112 & 2.72 & 277.00259 & 2.81 \\
\hline 226.98339 & 3.80 & 228.99913 & 65.12 \\
\hline 200.93470 & 3.82 & 226.98365 & 100.00 \\
\hline 182.92415 & 100.00 & 198.95566 & 14.36 \\
\hline \multirow[t]{4}{*}{151.96002} & 17.20 & 182.92439 & 33.95 \\
\hline & & 166.98361 & 8.52 \\
\hline & & 154.07769 & 99.37 \\
\hline & & 91.05440 & 14.34 \\
\hline $\begin{array}{l}\text { Fragment ion } \\
{[\mathrm{m} / \mathrm{z}]}\end{array}$ & Relative abundance [\%] & Fragment ion $[\mathrm{m} / \mathrm{z}]$ & Relative abundance \\
\hline \multicolumn{2}{|c|}{ 10-methyl-5H-phenarsazinine-10-sulfide } & & \\
\hline 289.99822 & 15.79 & & \\
\hline 241.99471 & 100.0 & & \\
\hline 168.08095 & 2.34 & & \\
\hline $\begin{array}{l}\text { Fragment ion } \\
{[\mathrm{m} / \mathrm{z}]}\end{array}$ & Relative abundance [\%] & Fragment ion $[\mathrm{m} / \mathrm{z}]$ & Relative abundance \\
\hline \multicolumn{2}{|c|}{ Dimethylphenylarsine sulfide } & \multicolumn{2}{|c|}{ Triphenylarsine sulfide } \\
\hline 214.98699 & 24.06 & 339.01804 & 1.24 \\
\hline 184.94008 & 10.48 & 303.01490 & 0.05 \\
\hline 182.00715 & 24.74 & 228.99910 & 8.67 \\
\hline 166.98366 & 100 & 226.98355 & 44.72 \\
\hline 136.94016 & 26.25 & 213.97569 & 0.76 \\
\hline 102.95235 & 2.15 & 182.92432 & 67.31 \\
\hline \multirow[t]{2}{*}{91.05444} & 11.13 & 154.07751 & 10.79 \\
\hline & & 151.96012 & 100 \\
\hline
\end{tabular}

MS/HRMS data for 10-hydroxy-5H-phenarsazinine-10-sulfide is not available due to disturbing high matrix background. 
FIgURE S-8 EXTRACTED ION CHROMATOGRAM FOR SEDIMENT SAMPLE \#8 CONTAINING SULFURCONTAINING PHENYLARSENIC CHEMICALS AND TARGET CHEMICALS

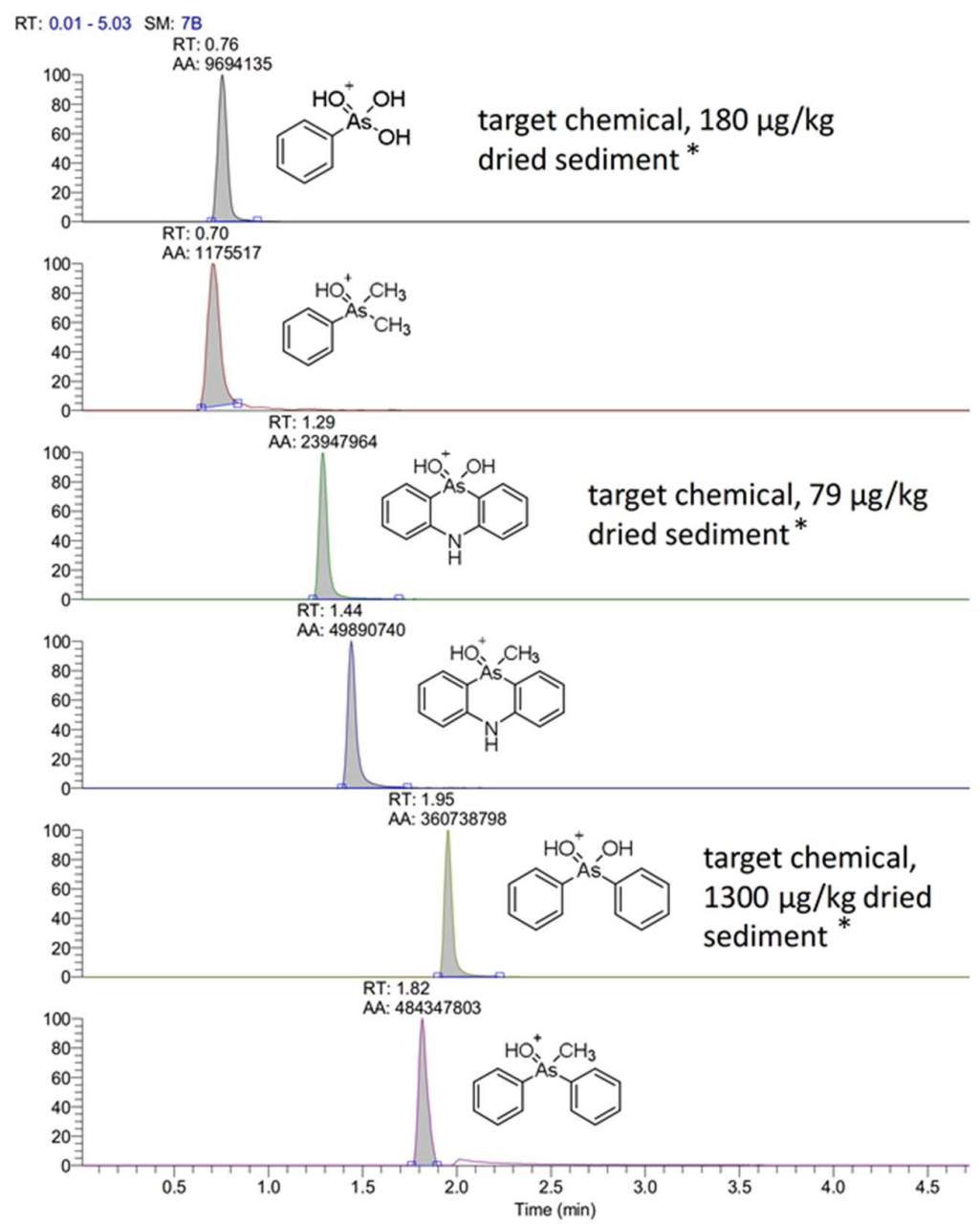

* Concentrations of target chemicals have been quantified previously using LC-MS/MS method from the same sediment sample. 
FIgURE S-9 EXTRACTED ION CHROMATOGRAM FOR SEDIMENT SAMPLE \#8 CONTAINING SULFURCONTAINING PHENYLARSENIC CHEMICAL AND TARGET CHEMICAL

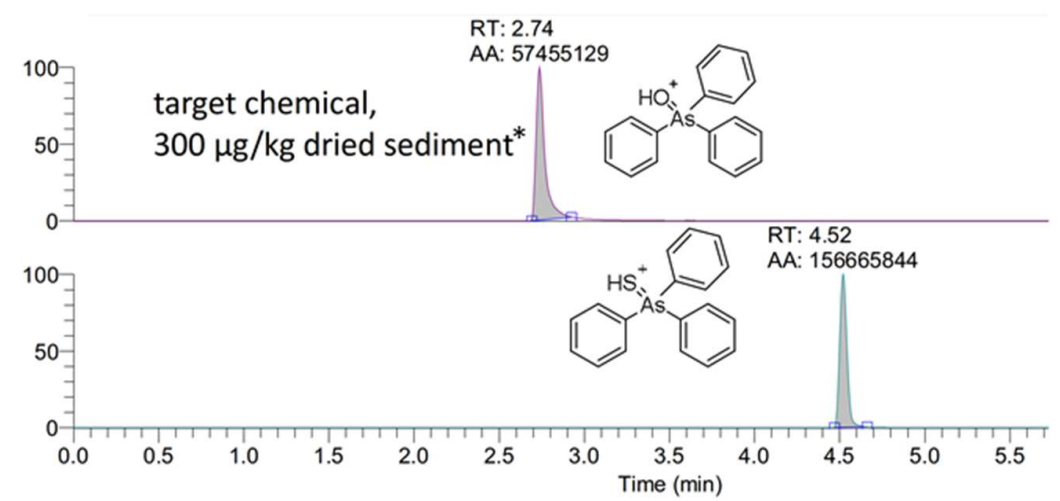

* Concentrations of target chemical have been quantified previously using LC-MS/MS method from the same sediment sample.

FIGURE S-10 SPECTRUM FOR HYDROXYPHENARSAZINIC ACID

M19100606 \#57 RT: 1.18 AV: 1 SB: 33 0.91-1.09, 1.24-1.61 NL: $1.52 E 4$

F: FTMS + CESI Full ms2 292.0000@hcd25.00 [70.0000-300.0000]

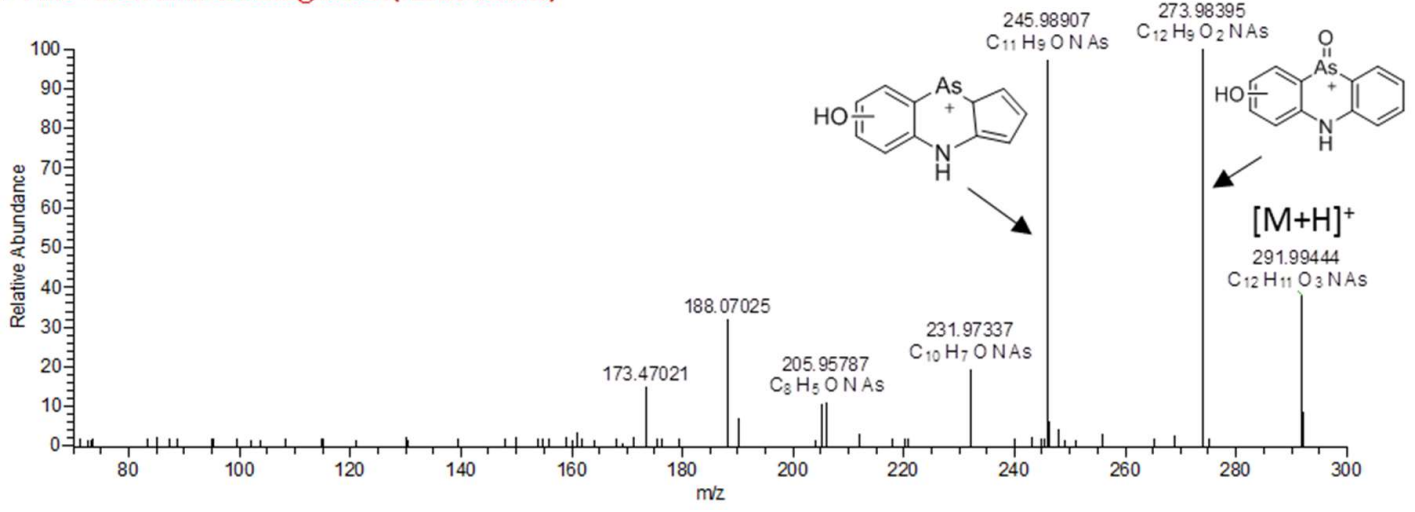


FIGURE S-11 EXTRACTED ION CHROMATOGRAM AND SPECTRA FOR TWO ISOMERS OF HYDROXYDIPHENYLARSINIC ACID

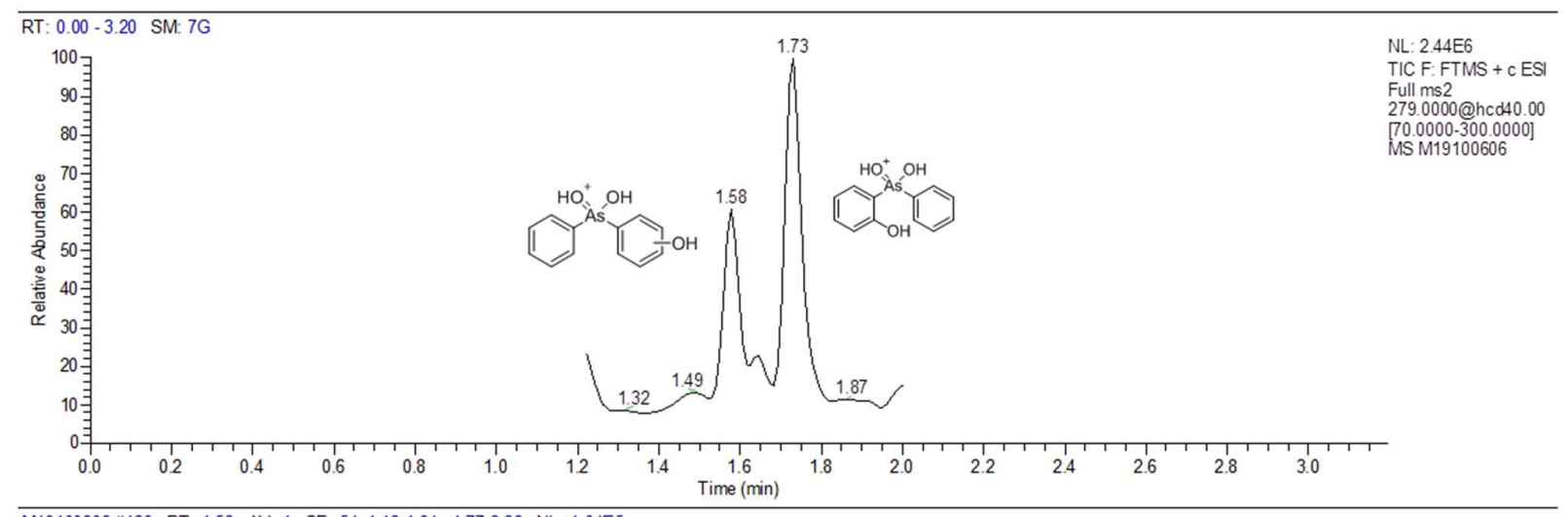

M19100606 \#139 RT: 1.58 AV: 1 SB: $541.12-1.64,1.77-2.26$ NL: $4.04 E 5$

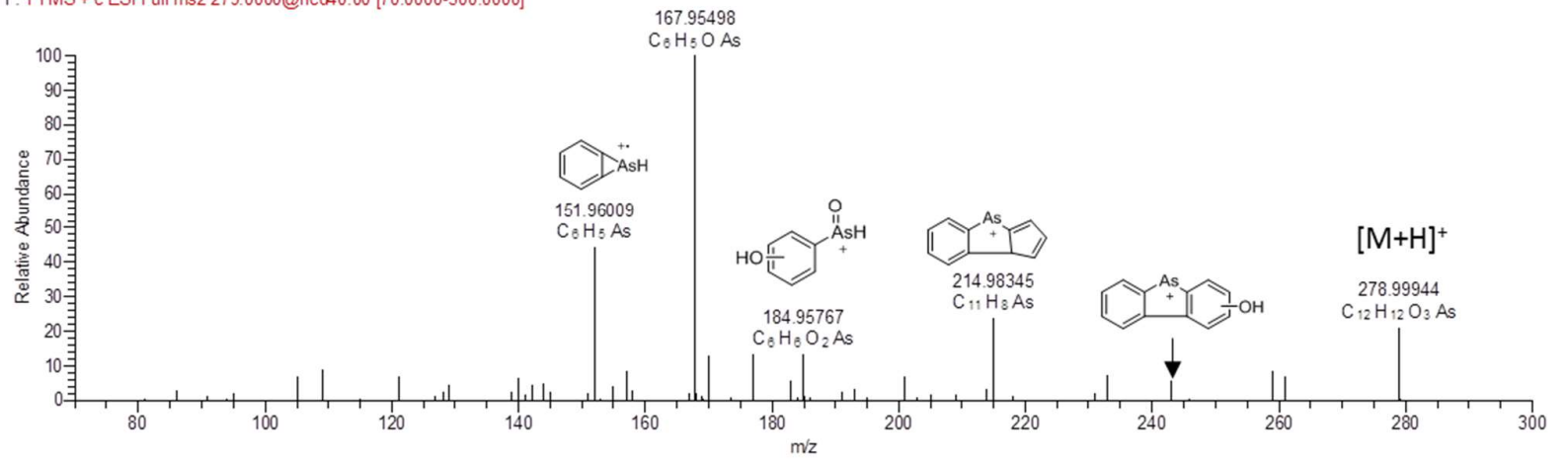

M19100606 \#171 RT: 1.73 AV: 1 SB: 54 1.12-1.64, 1.77-2.26 NL: $3.11 \mathrm{E} 5$

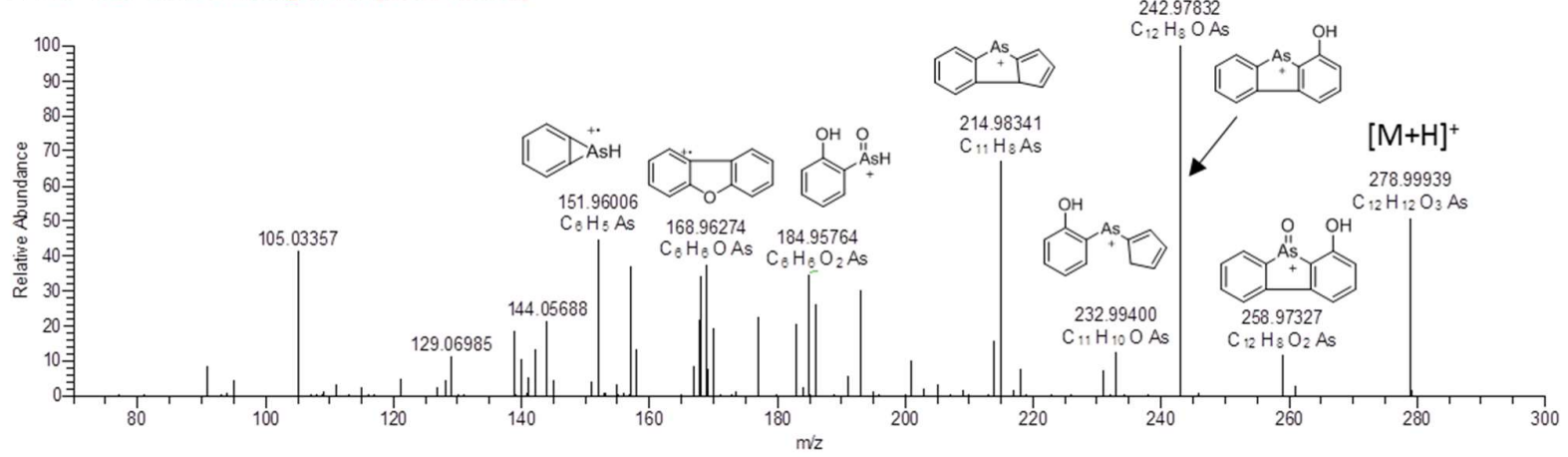


FIGURE S-12 EXTRACTED ION CHROMATOGRAM AND SPECTRA FOR TWO ISOMERS OF HYDROXYTRIPHENYLARSINE OXIDE
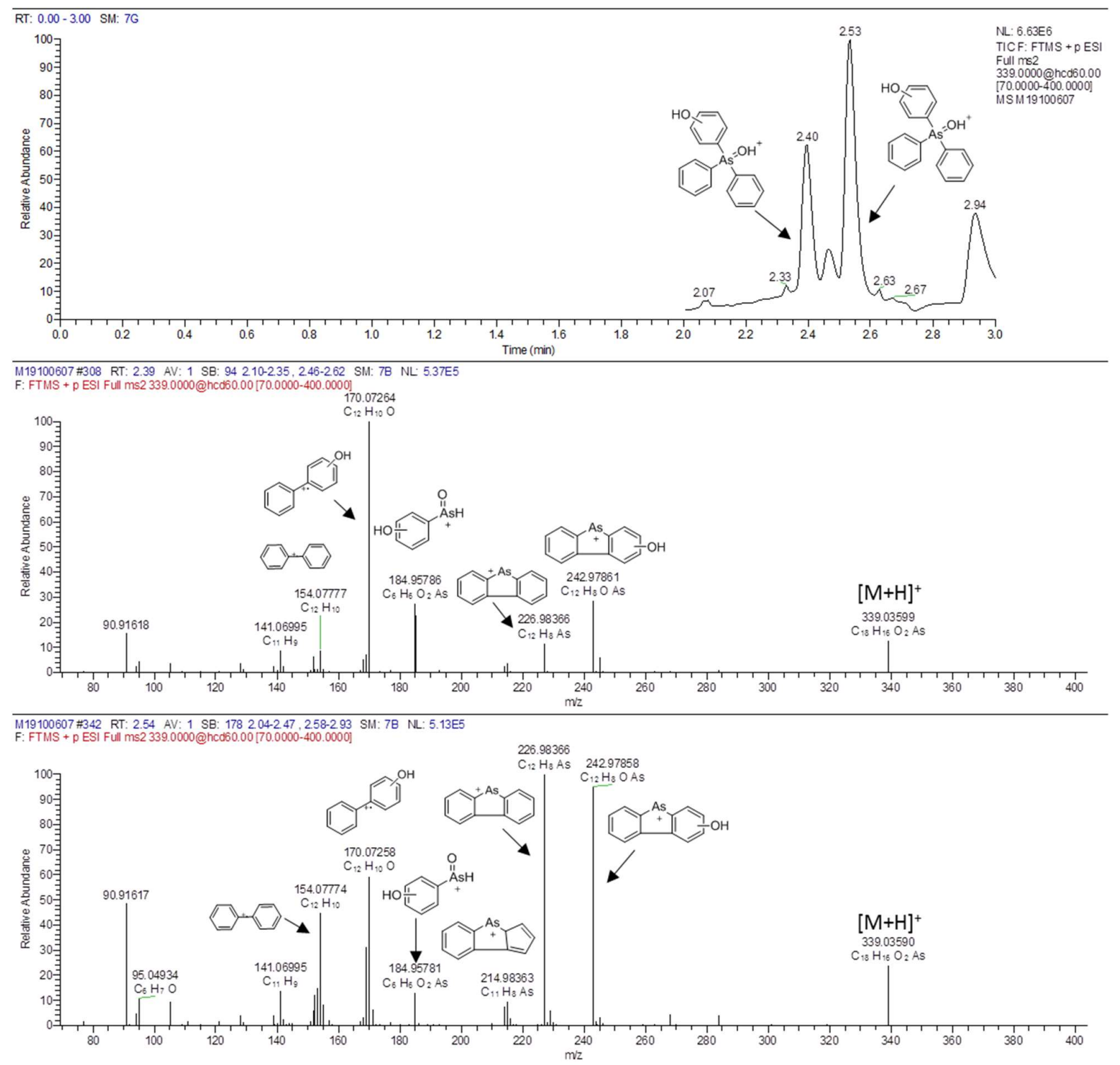